\title{
A REMARK ON EXPONENTIAL GROWTH AND THE SPECTRUM OF THE LAPLACIAN
}

\author{
Yusuke Higuchi ${ }^{1}$
}

\begin{abstract}
In terms of the exponential growth of a non-compact Riemannian manifold, we give an upper bounds for the bottom of the essential spectrum of the Laplacian. This is an improvement of Brooks' result.
\end{abstract}

\section{Introduction}

Let $M$ be a smooth, complete, non-compact Riemannian manifold, and $\Delta$ the Laplace-Beltrami operator on $L^{2}(M)$, where its sign is chosen so that it becomes a positive operator. We denote by $\lambda_{0}$ the bottom (that is, the greatest lower bound) of the spectrum of $\Delta$ and by $\lambda_{0}^{\text {ess }}$ the bottom of the essential spectrum. It is easy to see that $\lambda_{0} \leq \lambda_{0}^{\text {ess }}$, and that $\lambda_{0}^{\text {ess }}=\lim _{K} \lambda_{0}(M-K)$, where $K$ runs over an increasing set of compact subdomains of $M$ such that $\cup K=M$ and $\lambda_{0}(M-K)$ stands for the bottom of the spectrum of $\Delta$ with the Dirichlet boundary condition on $\partial K$. For a compact manifold, the essential spectrum is empty, thus we put $\lambda_{0}^{e s s}=\infty$.

There exist many works on the estimates for $\lambda_{0}$ or $\lambda_{0}^{\text {ess }}$ (for instance, [1], [2], [3], [4], [5], [7], [8]). Among them, R. Brooks ([2], [3]) has given the upper bounds for $\lambda_{0}^{\text {ess }}$ in terms of the volume growth: Pick a point $x_{0} \in M$ and let $B(r)$ be the ball of radius $r$ around $x_{0}$ and $V(r)$ the volume of this ball. It is shown that $\lambda_{0}^{e s s} \leq \bar{\mu}_{v}^{2} / 4$ if the volume of $M$ is infinite in [2] and that $\lambda_{0}^{e s s} \leq \bar{\mu}_{f}^{2} / 4$ if that is finite in [3], where $\bar{\mu}_{v}$ and $\bar{\mu}_{f}$ are the exponential volume growth of $M$, respectively, defined as

$$
\bar{\mu}_{v}=\limsup _{r \rightarrow \infty} \frac{1}{r} \log V(r) \quad \text { and } \quad \bar{\mu}_{f}=\limsup _{r \rightarrow \infty} \frac{-1}{r} \log (\operatorname{Vol}(M)-V(r)) .
$$

The purpose of this note is to give an estimate for $\lambda_{0}^{e s s}$ using another kind of exponential growth than Brooks'. This estimate is not only a slight improve-

1991 Mathematics Subject Classification: Primary 58G25, Secondary 35P15.

Key words and phrases: spectral geometry, growth function, essential spectrum.

${ }^{1}$ Partially supported by the Ministry of Education, Science, Sports and Culture of Japan under the Grant-in-Aid No. 09304022.

Received February 21, 2000; revised May 10, 2000. 
ment of Brooks' results but a continuous analogue of the result for the discrete Laplacian on an infinite graph ([6]).

Let us state our result. For the ball $B(r)=B\left(r, x_{0}\right)$ of radius $r$ whose origin is an arbitrary fixed point $x_{0} \in M$ and any fixed positive number $\delta$, we set

$$
B_{\delta}(\partial B(r))=\{x \in M-B(r) \mid \rho(x, \partial B(r)) \leq \delta\},
$$

where $\rho(x, \partial B(r))$ is the distance between $x$ and $\partial B(r)$, and denote by $S_{\delta}(r)$ the volume of $B_{\delta}(\partial B(r))$. Moreover, we set

$$
\mu_{0}=\lim _{\delta \rightarrow 0} \liminf _{r \rightarrow \infty} \mu_{\delta}(r) \text { and } \bar{\mu}_{0}=\lim _{\delta \rightarrow 0} \limsup _{r \rightarrow \infty} \mu_{\delta}(r),
$$

where $\mu_{\delta}(r)=(1 / r) \log S_{\delta}(r)$. Our result is the following:

THEOREM 1. For a non-compact manifold $M$, we have $\lambda_{0}^{e s s} \leq \mu^{2} / 4$, where $\mu=$ $\max \left(\mu_{0}, 0\right)$ if the volume of $M$ is infinite and $\mu=\bar{\mu}_{0}$ if that is finite.

We have $0 \leq \mu \leq \bar{\mu}_{v}$ in the infinite case and $\left|\bar{\mu}_{0}\right| \leq \bar{\mu}_{f}$ in the finite as is seen later. In this sense, Theorem 1 is somewhat better than Brooks'. The next corollary follows from Theorem 1 directly. We set

$$
\mu_{v}=\liminf _{r \rightarrow \infty} \frac{1}{r} \log V(r) \quad \text { and } \quad \mu_{f}=\liminf _{r \rightarrow \infty} \frac{-1}{r} \log (\operatorname{Vol}(M)-V(r)) .
$$

COROLlary 2. We have $\lambda_{0}^{e s s} \leq \mu_{v}^{2} / 4$ if the volume of $M$ is infinite, and $\lambda_{0}^{e s s} \leq$ $\mu_{f}^{2} / 4$ if that is finite.

The author would like to express his sincere gratitude to Professors Tomoyuki Shirai and Yoichiro Takahashi for their comments.

\section{Proof of Theorem 1}

For an arbitrary fixed $\delta>0$, we set

$$
\mu_{\delta}=\liminf _{r \rightarrow \infty} \mu_{\delta}(r) \quad \text { and } \quad \bar{\mu}_{\delta}=\limsup _{r \rightarrow \infty} \mu_{\delta}(r),
$$

where $\mu_{\delta}(r)=(1 / r) \log S_{\delta}(r)$. Theorem 1 follows from the following:

THEOREM 3. 1) If $M$ has infinite volume, then $\lambda_{0}^{e s s} \leq \mu_{\delta}^{2} / 4$ for any fixed $\delta>$ 0. Moreover, $\lambda_{0}^{e s s}=0$ if $\mu_{\delta}<0$.

2) If $M$ has finite volume, then $\lambda_{0}^{e s s} \leq \bar{\mu}_{\delta}^{2} / 4$ for any fixed $\delta>0$.

Proof of Theorem 1 and Corollary 2 from Theorem 3. We first assume that $M$ has infinite volume. It is obvious that $\mu_{\delta_{1}} \leq \mu_{\delta_{2}}$ if $\delta_{1}<\delta_{2}$. Then there exists $\mu_{0}=\lim _{\delta \rightarrow 0} \mu_{\delta}$, and we clearly have

$$
\mu_{0} \leq \mu_{\delta} \leq \mu_{v} \leq \bar{\mu}_{v} .
$$


Let $\mu_{0}<0$. Then, from the above, there exists $\delta>0$ such that $\mu_{\delta}<0$. Thus $\lambda_{0}^{\text {ess }}=0$.

Next, we assume that $M$ has finite volume. It holds also here that $\bar{\mu}_{\delta_{1}} \leq \bar{\mu}_{\delta_{2}}$ if $\delta_{1}<\delta_{2}$. Therefore there exist $\bar{\mu}_{0}=\lim _{\delta \rightarrow 0} \bar{\mu}_{\delta}$ and $\bar{\mu}_{\infty}=\lim _{\delta \rightarrow \infty} \bar{\mu}_{\delta}$; we have

$$
\bar{\mu}_{0} \leq \bar{\mu}_{\delta} \leq \bar{\mu}_{\infty} \leq \limsup _{r \rightarrow \infty} \frac{1}{r} \log (\operatorname{Vol}(M)-V(r))=-\mu_{f} \leq 0 .
$$

Let $\bar{\mu}_{\delta}<0$. In this case, for any $\varepsilon>0$ such that $\bar{\mu}_{\delta}+\varepsilon<0$, there exists $r_{0}$ such that, for any $r \geq r_{0},(1 / r) \log S_{\delta}(r)<\bar{\mu}_{\delta}+\varepsilon$. Then we get, for any $r \geq r_{0}$,

$$
\operatorname{Vol}(M)-V(r)=\sum_{k=0}^{\infty} S_{\delta}(r+k \delta) \leq \sum_{k=0}^{\infty} \exp \left(\left(\bar{\mu}_{\delta}+\varepsilon\right)(r+k \delta)\right) .
$$

Thus, we have

$$
\frac{1}{r} \log (\operatorname{Vol}(M)-V(r)) \leq \bar{\mu}_{\delta}+\varepsilon-\frac{1}{r} \log \left(1-\exp \left(\left(\bar{\mu}_{\delta}+\varepsilon\right) \delta\right)\right)
$$

and $-\mu_{f} \leq \bar{\mu}_{\delta}+\varepsilon$. Since we can select arbitrary small $\varepsilon>0,-\mu_{f} \leq \bar{\mu}_{\delta}$. Therefore, by $(2.3),-\mu_{f}=\bar{\mu}_{\delta}$; we have also $-\mu_{f}=\bar{\mu}_{\delta}$ for any $\delta>0$. Moreover, we easily get $\bar{\mu}_{\delta}=0$ for any $\delta$ and $\bar{\mu}_{\delta}=-\mu_{f}=0$ if $\bar{\mu}_{\delta}=0$ for some $\delta$. Consequently, we have $\bar{\mu}_{0}=\bar{\mu}_{\infty}=-\mu_{f}$ and $\left|\bar{\mu}_{0}\right|=\mu_{f} \leq \bar{\mu}_{f}$. Hence the proof is completed.

Remark 4. It is obvious that $\lim _{\delta \rightarrow 0} S_{\delta}(r) / \delta=S(r)$, where $S(r)=S\left(r, x_{0}\right)$ is the surface area of the distance sphere of radius $r$ at $x_{0}$. In addition, it is also obvious that $\lim \inf _{r \rightarrow \infty}(1 / r) \log \left(S_{\delta}(r) / \delta\right)=\mu_{\delta}$ and $\lim _{\sup _{r \rightarrow \infty}}(1 / r) \log \left(S_{\delta}(r) / \delta\right)$ $=\bar{\mu}_{\delta}$ for any fixed $\delta$. Note that, for any $r$ and any fixed $\delta>0$, there exists $q_{r, \delta} \in$ $(r, r+\delta)$ such that

$$
S\left(q_{r, \delta}\right)=(B(r+\delta)-B(r)) / \delta=S_{\delta}(r) / \delta .
$$

Then, setting $\mu_{s}=\lim \inf _{r \rightarrow \infty}(1 / r) \log S(r)$ and $\bar{\mu}_{s}=\lim \sup _{r \rightarrow \infty}(1 / r) \log S(r)$, we have $\mu_{s} \leq \mu_{0} \leq \bar{\mu}_{0} \leq \bar{\mu}_{s}$. When $\mu_{s}=\mu_{0}$ or $\bar{\mu}_{s}=\bar{\mu}_{0}$, we can substitute in Theorem $1 \mu_{s}$ or $\bar{\mu}_{s}$ for $\mu_{0}$ or $\bar{\mu}_{0}$, respectively.

Now let us prove Theorem 3 following an idea in [6] and Brooks' one in [2].

Proof of Theorem 3. Let $\lambda_{0}(M-K)$ be the bottom of the spectrum of $\Delta$ on $L^{2}(M-K)$ with the Dirichlet boundary condition on $\partial K$. It is well-known that

$$
\lambda_{0}(M-K)=\inf \frac{\int_{M}\|\operatorname{grad} f\|^{2}}{\int_{M} f^{2}},
$$

where $f$ runs over uniformly Lipshitz functions with compact support on $M-K$. Then we only have to show the following: for any fixed $\delta>0$, for any compact subset $K$ and for any sufficiently small $\varepsilon, \varepsilon_{1}>0$, there exists a function $f$ supported in $M-K$ such that 


$$
\frac{\int_{M}\|\operatorname{grad} f\|^{2}}{\int_{M} f^{2}}<\alpha^{2}(\varepsilon)+\varepsilon_{1},
$$

where $\alpha(\varepsilon) \rightarrow \mu_{\delta} / 2$ as $\varepsilon \rightarrow 0$.

Consider a test function $f(x)=\exp (h(x)) \cdot \chi(x)$, where $\chi(x)$ has compact support in $M-K$. Then

$$
\begin{aligned}
\int_{M-K}\|\operatorname{grad} f\|^{2}= & \int_{M-K} e^{2 h(x)}\left(\|\operatorname{grad} h \cdot \chi+\operatorname{grad} \chi\|^{2}\right) \\
\leq & \int_{M-K} e^{2 h(x)}\left(2 \chi \cdot\langle\operatorname{grad} h, \operatorname{grad} \chi\rangle+\|\operatorname{grad} \chi\|^{2}\right) \\
& +\int_{M-K} f^{2}\|\operatorname{grad} h\|^{2} .
\end{aligned}
$$

For $x \in M$, let $\rho(x)=\rho\left(x, x_{0}\right)$ denote the distance from a fixed point $x_{0} \in M$. For $r$ sufficiently large so that $K \subset B(r-\delta)$, we set $\chi$ as follows:

$$
\chi(x)=\chi_{r}(x)= \begin{cases}0, & \text { if } x \in K \text { or } \rho(x)>r+\delta, \\ \rho(x, K) / \delta, & \text { if } 0<\rho(x, K) \leq \delta, \\ 1-\rho(x, B(r)) / \delta, & \text { if } r \leq \rho(x) \leq r+\delta, \\ 1, & \text { otherwise. }\end{cases}
$$

Then $\operatorname{grad} \chi$ is supported in $B_{\delta}(\partial B(r))$ and a neibourhood $B_{\delta}(\partial K)$ of radius $\delta$ about $\partial K$; moreover, $\|\operatorname{grad} \chi\| \leq 1 / \delta$. In addition, we put, for a fixed number $\alpha \geq 0$ and for a positive integer $j$,

$$
h_{j}(x)= \begin{cases}\alpha \rho(x), & \text { if } \rho(x) \leq j, \\ 2 \alpha j-\alpha \rho(x), & \text { if } \rho(x)>j .\end{cases}
$$

Note that, for every $j,\left\|\operatorname{grad} h_{j}\right\|^{2} \leq \alpha^{2}$, and that $h_{j}$ increases pointwise to $h=\alpha \rho$. Thus, for $r$ and $j$ sufficiently large and $r>j$, we have

$$
\int_{M-K}\|\operatorname{grad} f\|^{2} \leq \alpha^{2} \int_{M-K} f^{2}+\left(2 \alpha / \delta+1 / \delta^{2}\right)\left(\int_{B_{\delta}(\partial K)} e^{2 h_{j}}+\int_{B_{\delta}(\partial B(r))} e^{2 h_{j}}\right)
$$

and there exists a finite constant $C$ independent of $r$ and $j$ such that

$$
\left(2 \alpha / \delta+1 / \delta^{2}\right) \int_{B_{\delta}(\partial K)} e^{2 h_{j}} \leq C .
$$

From here, we divide our proof into two cases: the case of $M$ with infinite volume and that of $M$ with finite volume.

First, we assume that $M$ has infinite volume. Then it is obvious that

$$
\int_{M-K} f^{2}=\int_{M-K} e^{2 h_{j}} \chi_{r}^{2} \rightarrow \infty \quad \text { as } r, j \rightarrow \infty .
$$


Let $\mu_{\delta} \geq 0$. It follows form the definition of $\mu_{\delta}$ that, for any $\varepsilon>0$, there exists a sequence $\left\{r_{n}\right\}$ such that

$$
\mu_{\delta}\left(r_{n}\right)=\frac{1}{r_{n}} \log S_{\delta}\left(r_{n}\right) \leq \mu_{\delta}+\varepsilon
$$

and $r_{n}>2 j\left(2+\mu_{\delta} / \varepsilon\right)$ for every $n$. Therefore, setting $\alpha=\alpha(\varepsilon)=\left(\mu_{\delta}+2 \varepsilon\right) / 2$, we have

$$
\int_{B_{\delta}\left(\partial B\left(r_{n}\right)\right)} e^{2 h_{j}} \leq \exp \left(\left(2 j-r_{n}\right)\left(\mu_{\delta}+2 \varepsilon\right)\right) \cdot \exp \left(\left(\mu_{\delta}+\varepsilon\right) r_{n}\right) \leq 1 .
$$

By (2.9), (2.10), (2.11) and (2.13), we can select $n$ and $j$ such that

$$
\frac{\int_{M}\left\|\operatorname{grad} f_{n}\right\|^{2}}{\int_{M} f_{n}^{2}} \leq \alpha^{2}(\varepsilon)+\varepsilon_{1}
$$

for any $\varepsilon_{1}>0$, where $f_{n}=e^{h_{j}} \chi_{r_{n}}$.

If $\mu_{\delta}<0$, then, for any $\varepsilon>0$ satisfying $\mu_{\delta}+\varepsilon<0$, there exists a sequence $\left\{r_{n}\right\}$ such that $\mu_{\delta}\left(r_{n}\right) \leq \mu_{\delta}+\varepsilon$. Setting $\alpha=0$, that is, $\exp \left(h_{j}(x)\right)=\mathbf{1}$, we have

$$
\int_{B_{\delta}\left(\partial B\left(r_{n}\right)\right)} e^{2 h_{j}}=S_{\delta}\left(r_{n}\right) \leq \exp \left(\left(\mu_{\delta}+\varepsilon\right) r_{n}\right)<1 .
$$

Thus, for any $\varepsilon_{1}>0$, we can select $n$ such that $\int_{M}\left\|\operatorname{grad} f_{n}\right\|^{2} / \int_{M} f_{n}^{2} \leq \varepsilon_{1}$, where $f_{n}=\mathbf{1} \chi_{r_{n}}$. We finish the proof in the case of infinite volume.

Next, let $M$ have finite volume. Then, we clearly have $-\infty \leq \bar{\mu}_{\delta} \leq 0$; we may assume $-\infty<\bar{\mu}_{\delta} \leq 0$. It follows form the definition of $\bar{\mu}_{\delta}$ that, for any sufficiently small $\varepsilon>0$, there exists a sequence $\left\{r_{n}\right\}$ such that

$$
\bar{\mu}_{\delta}-\varepsilon \leq \mu_{\delta}\left(r_{n}\right)=\frac{1}{r_{n}} \log S_{\delta}\left(r_{n}\right) \leq \bar{\mu}_{\delta}+\varepsilon
$$

and $r_{n}>2 j\left(2 \varepsilon-\bar{\mu}_{\delta}\right) /\left(\varepsilon-2 \bar{\mu}_{\delta}\right)$ for every $n$. Here we can assume this sequence $\left\{r_{n}\right\}$ satisfies $r_{n+1}-r_{n} \geq \delta$ for any $n$. Setting $\alpha=\alpha(\varepsilon)=-\left(\bar{\mu}_{\delta}-2 \varepsilon\right) / 2$ and $g(x)=$ $e^{\alpha \rho(x)}$, we have

$$
\int_{B(r)} g^{2} \geq \sum_{A(r)} \int_{B\left(r_{n}+\delta\right)-B\left(r_{n}\right)} g^{2} \geq \sum_{A(r)} \exp \left(2 \alpha r_{n}\right) \cdot S_{\delta}\left(r_{n}\right) \geq \sum_{A(r)} \exp \left(\varepsilon r_{n}\right) \rightarrow \infty
$$

as $r \rightarrow \infty$, where $A(r)=\left\{n \mid r_{n}+\delta \leq r\right\}$. Then we have

$$
\int_{M-K} f^{2}=\int_{M-K} e^{2 h_{j}} \chi_{r}^{2} \geq \int_{B(j)} g^{2}-\int_{K} g^{2} \rightarrow \infty \quad \text { as } r, j \rightarrow \infty
$$

and

$$
\int_{B_{\delta}\left(\partial B\left(r_{n}\right)\right)} e^{2 h_{j}} \leq \exp \left(\left(2 j-r_{n}\right)\left(2 \varepsilon-\bar{\mu}_{\delta}\right)\right) \cdot \exp \left(\left(\bar{\mu}_{\delta}+\varepsilon\right) r_{n}\right) \leq 1
$$


In the same way as in the case of infinite volume, selecting sufficiently large $n$ and $j$, one obtain the desired estimate.

\section{REFERENCES}

[1] R. Brooks, Exponential growth and the spectrum of the Laplacian, Proc. Amer. Math. Soc., 82 (1981), 473-477.

[2] R. Brooks, A relation between growth and the spectrum of the Laplacian, Math. Z., 178 (1981), 501-508.

[ 3 ] R. Brooks, On the spectrum of noncompact manifolds with finite volume, Math. Z., 187 (1984), 425-432.

[4] J. CheEger, A lower bound for the smallest eigenvalue of the Laplacian, Problems in Analysis, A Symposium in honor of S. Bochner, Princeton Univ. Press, 1970, 195-199.

[ 5] H. Donnely, On the essential spectrum of a complete Riemannian manifold, Topology, 20 (1981), 1-14.

[6] Yu. Higuchi, Boundary area growth and the spectrum of discrete Laplacian, preprint.

[7] M. Pinsky, The spectrum of the Laplacian on a manifold of negative curvature I, J. Differential Geom., 13 (1978), 87-91.

[ 8 ] H. Urakawa, Spectra of Riemannian manifolds without focal points, Geometry of Manifolds (K. Shiohama ed.), Perspect. Math. 8, Academic Press, 1989, 435-443.

Mathematics Laboratories

COLlege of ARTS AND SCIENCES

SHOWA UNIVERSITY

4562 Kamiyoshida, Fujiyoshida

YAMANASHI, 403-0005, JAPAN

E-mail: higuchi@cas.showa-u.ac.jp 